\title{
Influence of visually induced expectation on perceived motor effort: A visual-proprioceptive interaction at the Santa Cruz Mystery Spot
}

\author{
BRUCE BRIDGEMAN \\ University of California, Santa Cruz, California
}

\begin{abstract}
It is known that dense objects seem heavier than larger, less dense objects of the same weight. We have investigated a related illusion, in which visual context biases the apparent weight of a single object. The apparatus is a cabin on a steep hillside near Santa Cruz, CA, tilted $17^{\circ}$ from vertical. From its ceiling hangs a weight on a chain. The cabin's tilt makes the weight appear suspended at an angle. Pushing the weight toward the visually based vertical is perceived as difficult, whereas pushing it away from the visual vertical is perceived as easy. Seven subjects pushed the weight in both directions, judging required effort on a double-anchored 1-10 scale. All experienced the effort illusion, with no significant subject effect. When subjects' eyes were closed, the effect was smaller but still present. Apparently proprioceptive and skin inputs, equal for both directions, are ignored or underweighted as visually based expectations influence perceived effort.
\end{abstract}

Normally, humans are sensitive to the effort required of them as they perform a motor task; lifting heavy weights requires more effort than does lifting light ones, and heavy weights exert more pressure on the lifting organ (fingers, hand, etc.) than do light ones. The motor system prepares for the expected difference in required motor effort by evaluating the probable weight of an object and appropriately adjusting grip force and load force (proportional to the initial innervation strength of lifting muscles) before the physical lifting begins (Johansson \& Westling, 1984, 1988). Nonvisual sources of sensory information for making estimates of the weight of an object include (1) proprioception from the muscles; (2) comparisons of muscle efference required to execute a lifting motion with the speed, acceleration, and amplitude of the resulting movement; and (3) pressure and shear causing deformations of the skin surfaces in contact with the object.

In terms of physiology, this list is exhaustive; but in practice, other factors influence perceived weight as well. Perceived weight has been studied extensively in the size-weight illusion, first described by Charpentier (1891). If two objects of different size but equal weight are lifted, the smaller object will be judged heavier. Motor parameters adapt to this situation within a few trials while the perceptual illusion persists (Flanagan \& Beltzner, 2000), an example of a dissociation between cognitive and sensorimotor function that has also been demonstrated in other contexts (Bridgeman, Gemmer, Forsman, $\&$ Huemer, 2000; Milner \& Goodale, 1995). The role of

The author thanks the management of the Santa Cruz Mystery Spot for their generous cooperation. Correspondence concerning this article should be addressed to B. Bridgeman, Department of Psychology, University of California, Santa Cruz, CA 95060 (e-mail: bruceb@cats.ucsc.edu). experience with objects in the origin of the illusion is demonstrated in developmental experiments, where the visual effect increases with age (Pick \& Pick, 1967).

The size-weight illusion is important to psychology, because it demonstrates that expectations and assumptions are as important to perceived weight as the actual effort required to move objects. The illusion is affected by many factors, however, including the relative extents and volumes of the two objects, their textures, their locations, and haptic versus visual cues (Ellis \& Lederman, 1993). In the present study, a variation of the illusion is introduced in which both low- and high-effort actions are directed toward the same object, with all of these potential confounds controlled in one step. The new design opens the way for a more quantitative assessment of the illusion and of the factors that affect it.

\section{METHOD}

\begin{abstract}
Apparatus
The experiment was conducted in a tilted cabin at the Mystery Spot in Santa Cruz, CA, a tourist attraction in which visitors experience the strong perceptual effects of a tilted environment. Although Mystery Spot guided tours include a colorful story about magnetic anomalies and gravitational deviations, the effects are well understood (Shimamura \& Prinzmetal, 1999).

Built for the purpose of creating a visual illusion, the cabin is tilted $17^{\circ}$ from vertical (Figure 1). The tilt angle is not orthogonal to the walls of the cabin, so that the experiment room of the cabin has one corner lower than the others. At this location, a triangular segment of level floor (perpendicular to the gravity vector) fills the corner, although the level region appears to be tilted upward toward the corner. Above the level floor segment, an $8-\mathrm{kg}$ weight is suspended from the ceiling on a $1.2 \mathrm{-m}$ chain, appearing to hang at an angle, although of course the chain actually follows the gravity vector. The weight is a solid, spherical metal ball.
\end{abstract}



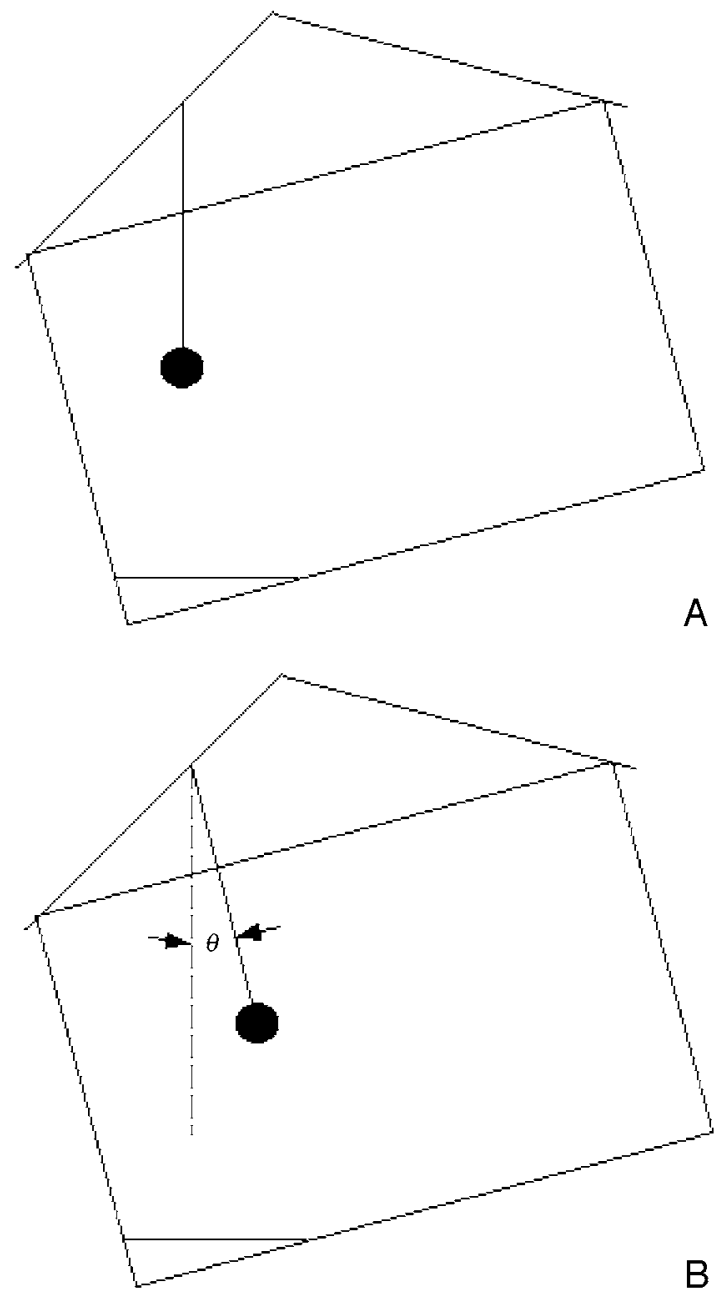

Figure 1. Cross-sections of the experiment cabin at the Santa Cruz Mystery Spot. (A) The weight (solid black circle) is hanging passively in the gravitational vertical. The floor at the lower left corner of the image is objectively horizontal. (B) An observer has pushed the weight through the angle $\theta$ into the visual vertical, with the chain parallel to the cabin walls. The dashed line represents a gravity vector with its origin at the anchor point of the chain. The apparent vertical is between the visual vertical and the gravitational vertical. Image B appears misaligned to the left of Image A but is actually aligned with it, creating another visual illusion.

\section{Procedure}

Observers were tested individually with a magnitude estimation technique. They were given a 10 -value double-anchored scale with which to judge the effort required in moving the weight, where 1 was described as effortless and 10 was immovable, fixed. Since the observers clearly would not use either of these anchor values, the measure reduced to an 8-value scale with double out-of-range anchors. The observers stood directly in front of the weight on the objectively level portion of the floor, so that pushes to one side and the other would have the same geometry and would share a similar level of difficulty. Half of the observers were asked to push the weight toward the corner and then away from the corner, giving a separate effort estimation for each direction. The other half of the observers were asked to perform the same actions in the reverse order. Because motor compensation for illusions of effort degrades quickly (Flanagan \& Beltzner, 2000), the observers made only one estimate of effort for each direction, although they were not timed and were allowed to move the weight as much as they desired before giving an estimate. They pushed on the side of the ball, without lifting it.

Because the actual force applied to the weight depends on the deviation of the ball from vertical, the observers' task was really to estimate the parameter $M$ (mass) in the equation

$$
F=M \cdot \sin \theta \cdot g
$$

where $F$ is the force applied, $\theta$ is the angle of deviation of the chain from gravitational vertical, and $g$ is the accelerational gravity constant. Because $g$ is constant, mass and weight will be used interchangeably here. The critical parameter, then, is not the force that the observer applies to the weight but the relationship between $F$ and $M$. Minor variations in the actual forces applied and the amplitudes of the movements have no significant effect on the weight estimate, as the results show.

\section{Subjects}

The observers were 7 undergraduate and graduate student volunteers from the University of California, Santa Cruz. All gave informed consent to participate in the study.

\section{RESULTS}

Perceived effort in pushing toward visual vertical was significantly greater than perceived effort in pushing away from visual vertical in all observers (Figure 2), the size of the effect averaging 2.9 magnitude estimation units $\left(t_{6}=4.05, p=.0067\right)$. There was no significant subject effect, either in pushing toward vertical $[F(1,5)=$ $2.27, p=.192]$ or in pushing away from vertical $[F(1,5)=$ $0.66, p=.45]$.

All observers were given an opportunity to express their open-ended reactions to the experiment during debriefing. All but 1 or 2 of the observers indicated that they were surprised by the difference in effort required to push the weight in the two directions.

In 2 of the observers, the experiment was repeated with eyes closed during the entire protocol (pushing the weight in both directions). They were instructed to close their eyes immediately before touching the weight, after positioning themselves before it, and to keep their eyes closed until they had made magnitude estimations for both directions. The procedures were otherwise the same as during the eyes-open experiment. The magnitude of the contrast effect was smaller, 1.0 magnitude estimation units in each observer, in the same direction as in the main experiment.

\section{DISCUSSION}

The illusion of perceived weight found in this experiment is thought to depend on a mismatch between expected and actual weight. The expected effort required in order to move the pendulum weight was based on moving the pendulum away from the illusory vertical position, not the true rest position.

Because of a consistent tilt illusion that results in a deviation of the perceived vertical from the gravitational vertical in the Mystery Spot (Shimamura \& Prinzmetal, $1999)$, we can define an illusory angle of deviation $\theta_{\mathrm{I}}$ 


\section{Perceived Effort}

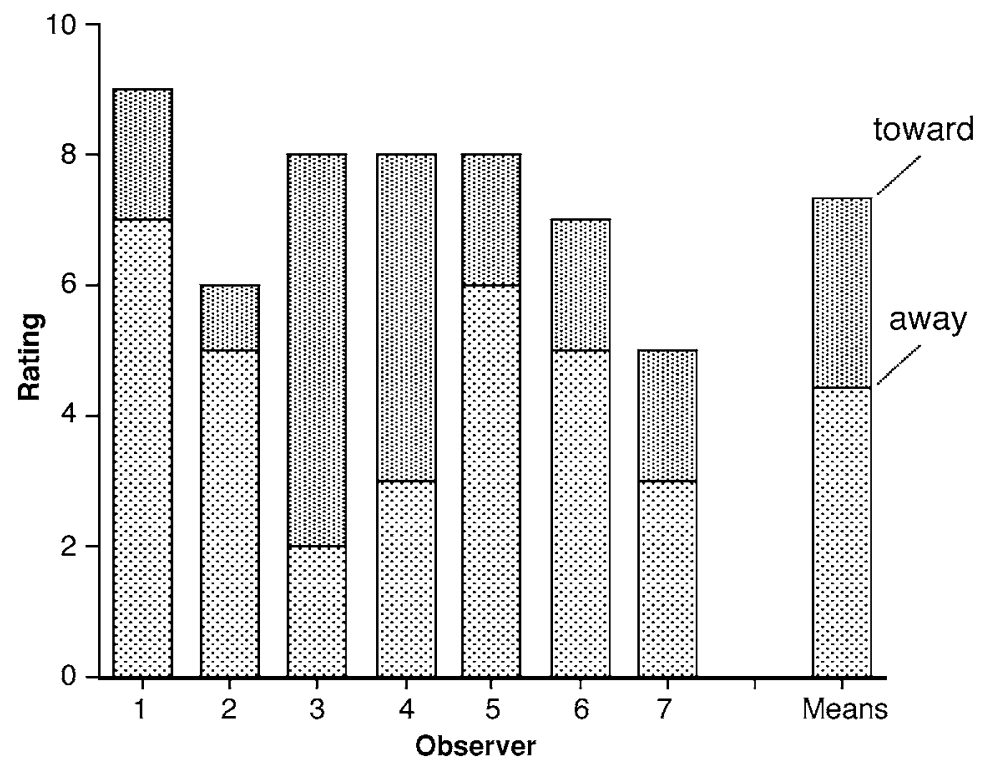

Figure 2. Breakdown of perceived effort by observer. "Toward" means effort at pushing the weight toward the visual vertical orientation. "Away" means effort at pushing the weight away from the visual vertical orientation. The rightmost column shows perceived effort in moving the test weight toward and away from the visual vertical, averaged across subjects.

that will distort the expected force that must be applied to move the weight. For motion toward the apparent vertical, the expected force $F_{\text {exp }}$ is

$$
F_{\text {exp }}=M \cdot \sin \left(\theta-\theta_{\mathrm{I}}\right) \cdot g
$$

whereas the expected force away from the apparent vertical is

$$
F_{\text {exp }}=M \cdot \sin \left(\theta+\theta_{\mathrm{I}}\right) \cdot g .
$$

In Equation 2, $\left(\theta-\theta_{\mathrm{I}}\right)$ is negative for small values of $\theta$, so that the pendulum appears to be deviated from what should be its resting position. One should expect a negative force (a push in the opposite direction) to deviate the pendulum weight into the apparent vertical. At rest, the weight appears suspended in an impossible position, so that it should fall spontaneously toward the visual vertical. It is as though some additional force holds the weight in this unstable position. This is why the visual illusion is so compelling and why the expected force applied is so dramatically different from the actual force needed to move the weight. The expected and actual effects are in opposite directions.

In the case of pushing the pendulum away from the apparent vertical, the required force will always be less than the expected force $F_{\text {exp }}$, since $\sin \theta$ will always be less than $\sin \left(\theta+\theta_{\mathrm{I}}\right)$ within the range of angles used in the experiment.

Robust presence of the single-object weight illusion in all observers shows that differences in the relative volumes of two objects, assumed relative density, and so forth, are not essential to eliciting the size-weight illu- sion. Counterintuitively, perceived weight of the test object was not dependent only on the finger pressure and muscle proprioception required to move the weight, but rather on the difference between the expected force and the actual force required. The signals from the physiological sensors were not available to perception in an undistorted manner, because identical signals gave different perceptions. These signals were of course equal in the two movement conditions.

It remains to be seen whether muscle proprioception, efference copy, or tactile stimulation is responsible for the sensory feedback specifying the weight of the ball. The present design is ideal for identifying such an interaction, because vision has a strong effect on touch perception when a visual stimulus is presented near the hand but has a much weaker effect when the visual stimulus and the hand are spatially separated (Làdavas, Farnè, Zeloni, \& di Pellegrino, 2000). A deafferented subject, deprived of large sensory myelinated fibers from the nose down, could discriminate weights as well as normal controls (Fleury et al., 1995), presumably by comparing muscular efference copy with vision of the weights' motions. In this subject, limb proprioception was compromised. Since the quality of proprioceptive information for limb position is poor (Haggard, Newman, Blundell, \& Andrew, 2000), it probably did not contribute significantly to the discrimination. From this literature, one can infer that vision in the present experiment overcame primarily tactile cues to create the weight illusion.

At the conclusion of these experiments, the rationale of the study was explained to the Mystery Spot management. 
They responded that someone else had already performed a similar study, and that the results were published in a book available in the gift shop (Vogt, 1996). Vogt measured the actual force required in order to move the ball, claiming that it took $58 \%$ more physical force to move the weight toward the visual vertical than away. He attributed his result to a "gravitational vortex," and also measured anomalies in the resonant frequency of a crystal in the region. The psychophysical effects are described and understood in the present article without postulating supernatural forces. Thus the present article is the first scientific description of the single-object weight illusion.

\section{REFERENCES}

Bridgeman, B., Gemmer, A., Forsman, T., \& Huemer, V. (2000). Processing spatial information in the sensorimotor branch of the visual system. Vision Research, 40, 3539-3552.

Charpentier, A. (1891). Analyse expérimentale de quelques éléments de la sensation de poids [Experimental analysis of some aspects of weight perception]. Archives de Physiologie Normale et Pathologique, 3, 122-135.

Ellis, R. R., \& Lederman, S. J. (1993). The role of haptic versus visual volume cues in the size-weight illusion. Perception \& Psychophysics, 53, 315-324.

Flanagan, J. R., \& Beltzner, M. A. (2000). Independence of perceptual and sensorimotor predictions in the size-weight illusion. $\mathrm{Na}$ ture Neuroscience, 3, 737-741.
Fleury, M., Bard, C., Teasdale, N., Paillard, J., Cole, J., Lajoie, Y., \& LAMARRE, Y. (1995). Weight judgment: The discrimination capacity of a deafferented subject. Brain, 118, 1149-1156.

Haggard, P., Newman, C., Blundell, J., \& Andrew, H. (2000). The perceived position of the hand in space. Perception \& Psychophysics, 68, 363-377.

Johansson, R. S., \& Westling, G. (1984). Roles of glabrous skin receptors and sensorimotor memory in automatic control of precision grip when lifting rougher or more slippery objects. Experimental Brain Research, 56, 550-564.

Johansson, R. S., \& Westling, G. (1988). Coordinated isometric muscle commands adequately and erroneously programmed for the weight during lifting task with precision grip. Experimental Brain Research, 71, 59-71.

Làdavas, E., Farnè, A., Zeloni, G., \& di Pellegrino, G. (2000). Seeing or not seeing where your hands are. Experimental Brain Research, 131, 458-467.

Milner, A. D., \& Goodale, M. A. (1995). The visual brain in action. Oxford: Oxford University Press.

PICK, H. L., JR., \& PICK, A. D. (1967). A developmental and analytic study of the size-weight illusion. Journal of Experimental Child Psychology, 5, 362-371.

Shimamura, A. P., \& Prinzmetal, W. (1999). The Mystery Spot illusion and its relation to other visual illusions. Psychological Science, 10, 501-507.

VoGT, D. B. (1996). Gravitational mystery spots of the United States: Explained using the theory of multidimensionality. Bellevue, WA: Vector Associates.

(Manuscript received October 3, 2002 ; revision accepted for publication January 27, 2003.) 